\title{
Racial dot maps based on dasymetrically modeled gridded population data
}

\author{
Anna Dmowska a and Tomasz Stepinski ${ }^{\mathrm{a}}$ \\ aspace Informatics Lab, Department of Geography and GIS, University of Cincinnati, OH, \\ USA
}

\begin{abstract}
Racial geography - mapping spatial distributions of different racial groups is of keen interest in a multiracial society like the United States. Racial dot map is a method of visualizing racial geography which depicts spatial distribution, population density, and racial mix in a single, easy-to-understand map. Because of the richness of information it carries, the dot map is an excellent tool for visual analysis of racial distribution. Presently used racial dot maps are based on the census data at the tract or the block level. In this paper, we present a method of constructing a more spatially accurate racial dot map based on a sub-block resolution population grid. The utility of our dot maps is further enhanced by placing dots on the map in random order regardless of the race they represent in order to achieve a more accurate depiction of local racial composition. We present a series of comparisons between dot maps based on tract, block, and grid data. The advantage of a grid-based dot map is evident from the visual comparison of all maps with an actual image of the mapped area. We make available the $\mathrm{R}$ code for constructing grid-based dot maps. We also make available 2010 grid-based racial dot maps for all counties in the conterminous United States.
\end{abstract}

\section{Introduction}

Residential racial segregation and racial diversity of population have been traditionally studied using a non-spatial statistical analysis with the results reported in the form of a table listing segregation/diversity indices (Farrell, 2008; Frey and Farley, 1996; Frey and Myers, 2005; Iceland, 2004; Iceland, Weinberg, and Steinmetz, 2002; Johnston, Poulsen, and Forrest, 2007; Lichter, Parisi, Grice, and Taquino, 2007; Logan, Stults, Farley, and Stults, 2004; Massey and Denton, 1987) for different metropolitan areas, counties etc. Such an approach did not require maps and, consequently, the issue of how best to map racial segregation and/or diversity had not arisen. However, more recently, demographers have turned their attention to spatial analysis (Bader and Warkentien, 2016; Clark, Anderson, Östh, and Malmberg, 2015; Farrell and Lee, 2011; Fasenfest, Booza, and Metzger, 2004; Holloway, Wright, and Ellis, 2012; Logan, 2015; Wong, 2015; Wright, Ellis, Holloway, and Wong, 2014) and the issue of how to design maps to best show the racial distribution of population became important.

In the United States, demographic data is released by the U.S. Census Bureau in the form of areal aggregates. Consequently, the overwhelming majority of maps illustrating Census data are choropleth map. In such maps, a study area is divided into census areal units (tracts, block groups, or blocks) and each unit is colored according to its value of a demographic variable. In racial demography two variables are most often used, one is a percentage of people of a given race (a numerical variable), and another is a diversity category that describes a character of a racial 
mix in a unit (a categorical variable). The first type of mapping requires several maps (one for each race) to communicate racial geography of the study area, whereas the second type communicates this racial geography in a single map. The second mapping method provides much more effective visualization of an overall racial character of the study area. Examples of maps showing racial diversity categories can be found in Bader and Warkentien (2016); Fasenfest et al. (2004); Holloway et al. (2012); Wright et al. (2014).

Figure 1 shows several adjoined census tracts in the city of San Fransisco centered around the Balboa Park. Panel A shows the map of the percentage of Asian population (maps of the percentage of other races would be needed to complete the information about racial character of this area). Three centrally located tracts have a strong majority Asian population, tracts to the south of the center have a smaller majority Asian population, and tracts to the north have even smaller, a non-majority percentage of Asians. Panel B shows a diversity/top race classification map. This map, which does not concentrate on Asians alone, but takes into consideration all races, provides more general information. Central and southern tracts have medium diversity population with Asians being the top contributor, the northern tracts have medium diversity population with whites being the top contributor, and one eastern tract has medium diversity population with Hispanics being the top contributor.

Figure 1C shows a racial dot map - a third type of map, that in recent years started to be used in segregation/diversity studies. Dot maps depict spatial distribution and density of discrete, labeled geographical objects (Kimerling, 2009; ?). In segregation/diversity studies the objects are people and the labels are their races. In Figure $1 \mathrm{C}$ the number of dots in a tract corresponds to its people count. Dots are colored in proportion to the racial composition and placed in random position within a tract. Such a map is arguably even better visualization of racial geography than the diversity map (Figure 1B) as it explicitly accounts for every person. Racial dot maps were popularized by two web applications enabling viewing such maps over the entire United States. The first map, from the University of Virginia (https://demographics.virginia.edu/DotMap/) is based on census block data, and another, from the New York Times (https://www.nytimes.com/interactive/2015/07/08/us/census-race-map.html), is based on census tracts. Racial dot maps have been shown to be a preferred tool in teaching about residential segregation (Seguin, Nierobisz, and Kozlowski, 2017).

Notice that maps in Figure 1 panels A, B, and C exhibit artifacts due to the fact that all three are based on census tracts. The maps show uniform properties (race percentage, diversity type, a pattern of dots) across the entire tract. In reality, tracts are not homogeneous. In addition to residential areas, they may contain uninhabited areas such as parks, highways, industrial and other non-residential buildings. As can be seen on the Google street map (Figure 1G) the site includes a park, a university, a high school, and the major highway, all of them unrecognized in panels A, B, and $\mathrm{C}$ as residentially uninhabited. The same problem remains even if smaller census units such as block groups or blocks are used instead of tracts. Another artifact is the abrupt change of racial character at the boundaries between tracts, this is especially noticeable in a dot map (panel C). These artifacts (and other problems, like, for example, incompatibility of census areas boundaries between different years) transcend segregation/diversity studies and applies to all census-based maps. They have been referred to by Sperling (2012) as "the tyranny of census geography".

To alleviate the "tyranny of census geography" in segregation/diversity studies we previously have developed (Dmowska and Stepinski, 2014, 2016, 2017a,b; Dmowska, Stepinski, and Netzel, 2017) high-resolution population grids for the entire conterminous United States. In such grid, each grid's cell contains density of all racial sub-populations at its location. Constructing race percentage maps and racial diversity type maps (equivalents of Figures $1 \mathrm{~A}$ and $1 \mathrm{~B}$, respectively) from the grid is straightforward; such maps for our example area in San Francisco are shown in Figures 1 panels D and E. An advantage of using gridded data instead of census-based data is immediately clear by comparing panels $\mathrm{A}$ with $\mathrm{D}$ and panels $\mathrm{B}$ with $\mathrm{E}$. The artifacts discusses above are not present in the grid-based maps. Also, the grid-based maps are more detailed, partially because they started from the census-block data and partially because the grid was constructed using dasymetric modeling (see the next section for details).

Racial dot map constructed from the population grid has the same advantage over the dot map constructed from the census tracts as the race percentage map and the racial diversity map do (see Figure $1 \mathrm{~F}$ ). However, constructing a dot map 

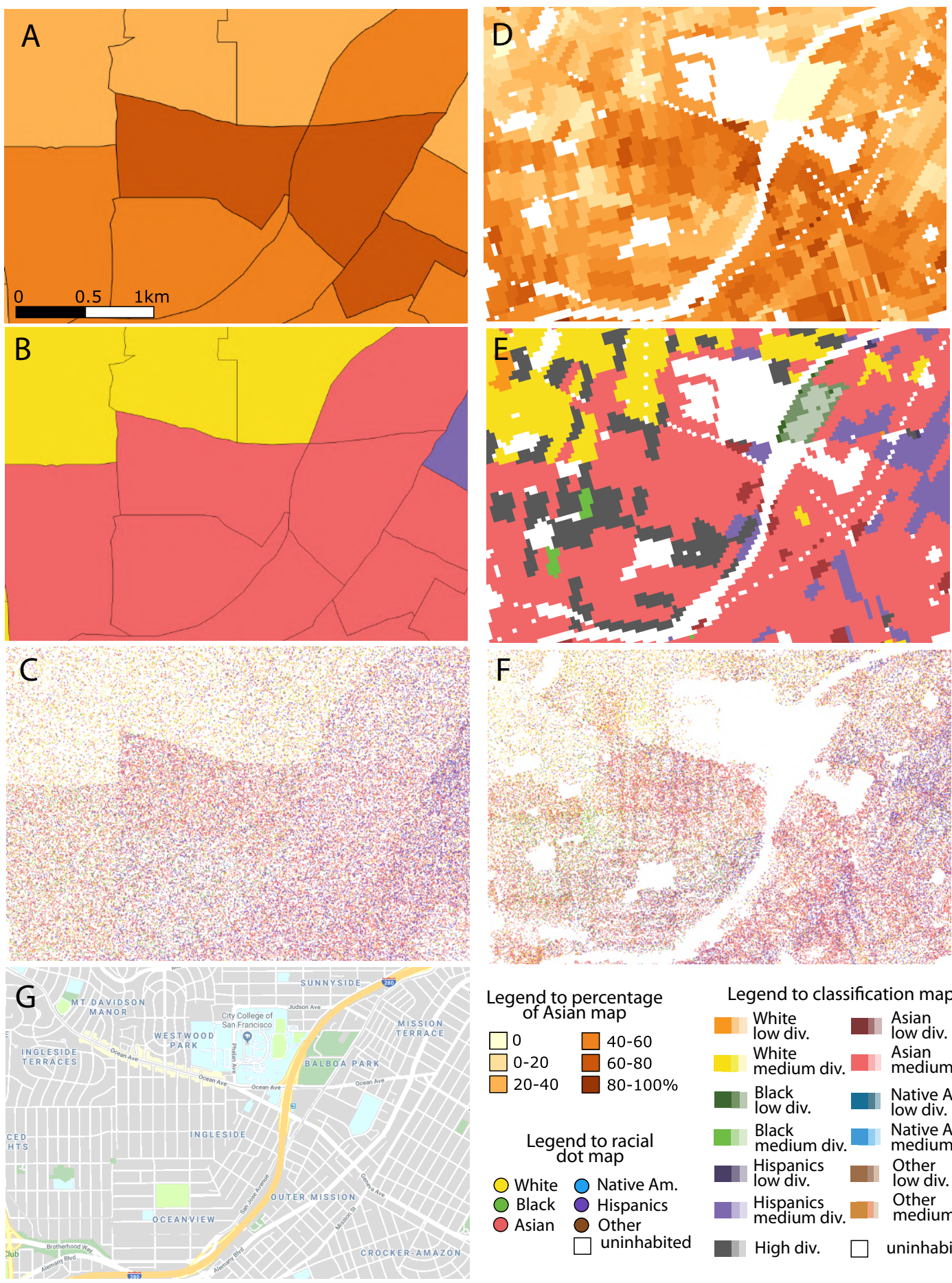

\section{Legend to classification map}

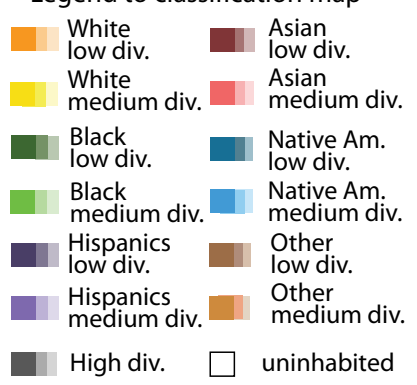

Figure 1. Comparison of different maps visualizing racial segregation/diversity in the Balboa Park site in San Francisco. Maps on the left, A, B, and C, are based on census tracts data and maps on the right, D, E, and $\mathrm{F}$, are based on gridded data. The top row shows maps depicting the percentage of the Asian population, the second row shows maps depicting diversity/top race classification, and the third row shows racial dot maps. Panel G is the Google street map of the site. Color shades in the legend for diversity classification maps reflect overall population density. 
from the high-resolution grid is not straightforward because grid stores data as a population density rather than as a people count. The major contribution of this paper is to present a method of constructing a racial dot map from high-resolution gridded data, to develop and to make publicly available the $\mathrm{R}$ code for grid-based dot maps, and to calculate and to make publicly available grid-based racial dot maps for all counties in the conterminous United States. We also present a series of examples in support of our claim that grid-based dot maps are more spatially accurate than tract or block-based dot maps. In these examples, all three types of dot maps are shown and their spatial accuracy can be visually assessed by comparison to an image of the mapped area.

\section{Methods}

This section is divided into two subsections. In the first subsection, we provide background information on gridded data; detailed information on gridded data is given in Dmowska and Stepinski (2017a); Dmowska et al. (2017). In the second subsection, we describe the construction of a racial dot map from the grid.

\subsection{Gridded data}

An input for our method of constructing grid-based racial dot maps consists of the population and racial grids available online through the SocScape web application (http://sil.uc.edu/webapps/socscape_usa/). They can also be downloaded by individual county and Metropolitan Statistical Area (MSA) from http://sil.uc.edu. Details of grid construction and its accuracy are described in Dmowska and Stepinski (2017a) and the benefits of using gridded population data over census aggregates are discussed in Dmowska et al. (2017).

The input data for grid construction is the U.S. Census Bureau block population data, the National Land Cover Dataset (NLCD) and the National Land Use Dataset (NLUD) (Theobald, 2014). The grid is constructed by redistribution of population in the block using dasymetric modeling (for a review of dasymetric modeling see Petrov (2012)) with LCLU and NLUD as ancillary data. LCLU and NLUD data have a significantly higher resolution (30m per cell) than a size of census blocks and can be used to guide redistribution of the within-block population due to the existence of a correlation between different categories of land cover/land use and different values of population density. This correlation and the derived dasymetric model are presented in Dmowska and Stepinski (2017a). The result is the grid of total population density (in units of people $/ 900 \mathrm{~m}^{2}$ ), with $900 \mathrm{~m}^{2}$ being an area of a single $(30 \mathrm{~m} \times 30 \mathrm{~m})$ grid cell. This resolution is dictated by the resolution of the LCLU and NLUD data. Note that a grid cell is not an aggregation unit, instead, it is a point in space for which a density of population is measured. Thus, $30 \mathrm{~m} \times 30 \mathrm{~m}$ is a nominal resolution of the population data in the form of the grid, not the actual resolution with which the locations of residences are known.

Dasymetric model distributes unevenly the total population of the block into its constituted cells in proportion to LCLU and NLUD categories of the cell. Thus, the cells having LCLU/NLUD categories associated with uninhabited areas will get no people, those associated with the low density of population will get a relatively small share of people, and those associated with the high density of population will get a relatively large share of people. The result is a sub-block spatial distribution of the population.

There is no high-resolution ancillary data that would correlate with the race of the population. Thus, to obtain population density for different races at a given cell we use relative ratios inherited from the block to which the cell belongs. Thus, sub-populations are not independently redistributed within a block, instead, they are redistributed in the same way as the total population. This still results in improved informational content of a grid over the block as people of all races are kept away from uninhabited areas and the population density is not constant across the block. In addition, using census blocks (which are much smaller than census tracts) as an input to the dasymetric model minimizes the possibility of different spatial distributions for different sub-populations.

We divide the total population into the following sub-populations: non-Hispanic 


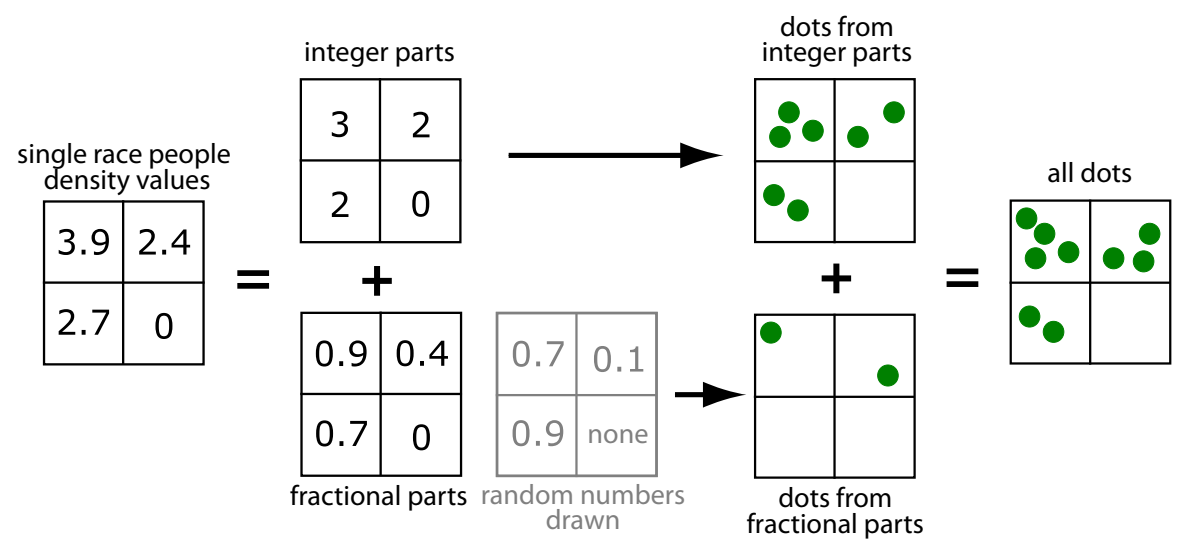

Figure 2. Illustration of the procedure to assign a given race dots to a grid cell. See the main text for explanation.

white, non-Hispanic black, non-Hispanic Asian, non-Hispanic American Indian, nonHispanic Native Hawaiian and Other Pacific Islander, non-Hispanic other race and Hispanic. Names of these sub-populations follow from the fact that census asks two different questions in its questionnaire, one about the race (white, black, Asian, Native Americans, Hawaiian/Pacific Islander, other), and another about ethnicity (Hispanic, non-Hispanic). For brevity we will use the following categories, White, Black, Hispanics, Asians, Native Americans, and Other, where Hawaiian/Pacific Islanders were incorporated into the Asian sub-population; we refer to these categories as races.

\subsection{Grid-based dot map}

To construct a dot map from gridded data we need to decide how many dots to put within each cell of a grid. The major issue is that the grid carries fractional numbers corresponding to a density of people rather than integer numbers corresponding to a count of people. Consider a cell with the overall density $D=D_{1}+\cdots+D_{6}$, where $D_{i}, i=1, \ldots 6$ are densities of different sub-populations present in the cell. We will describe the method for one person per dot map, but constructing a $k$-people per dot map is analogous except for starting with all densities divided by the same integer $k$.

Dots are assigned to the cell for each sub-population separately according to the procedure illustrated in Figure 2. In this figure, only a small portion of the grid consisting of four adjacent cells is shown with the numbers in each cell showing values of density $D_{i}$ for a given sub-population (say, for $i=2$, or the black subpopulation). Next, the value of density in each cell is decomposed into an integer part and a fractional part. The number of dots corresponding to a black sub-population in a cell is given by a sum of the integer part plus contribution from the fractional part that may be either one dot or zero dots. Whether it is one or zero is decided by a random number generator. A random number between 0 and 1 is generated. If the generated number is smaller than a fractional part the contribution is one dot, otherwise, it is zero dots. Thus, the probability of contributing an additional dot is proportional to the value of the fractional part; larger values are more likely to result in contributing an additional dot. With such procedure, the number of dots representing the black population in the entire census block is guaranteed to be close to an actual block count of this sub-population.

The procedure described above is repeated for all other sub-populations. Next, all dots are assigned random coordinates within a cell and a label (color) indicating which race they represent. The dots are drawn randomly without consideration of their label and placed on the map. The random order of placement minimizes the chances that dots representing a given race will be obscured by subsequently placed dots representing a different race.

Our method is implemented in R language R Core Team (2014). R is a com- 

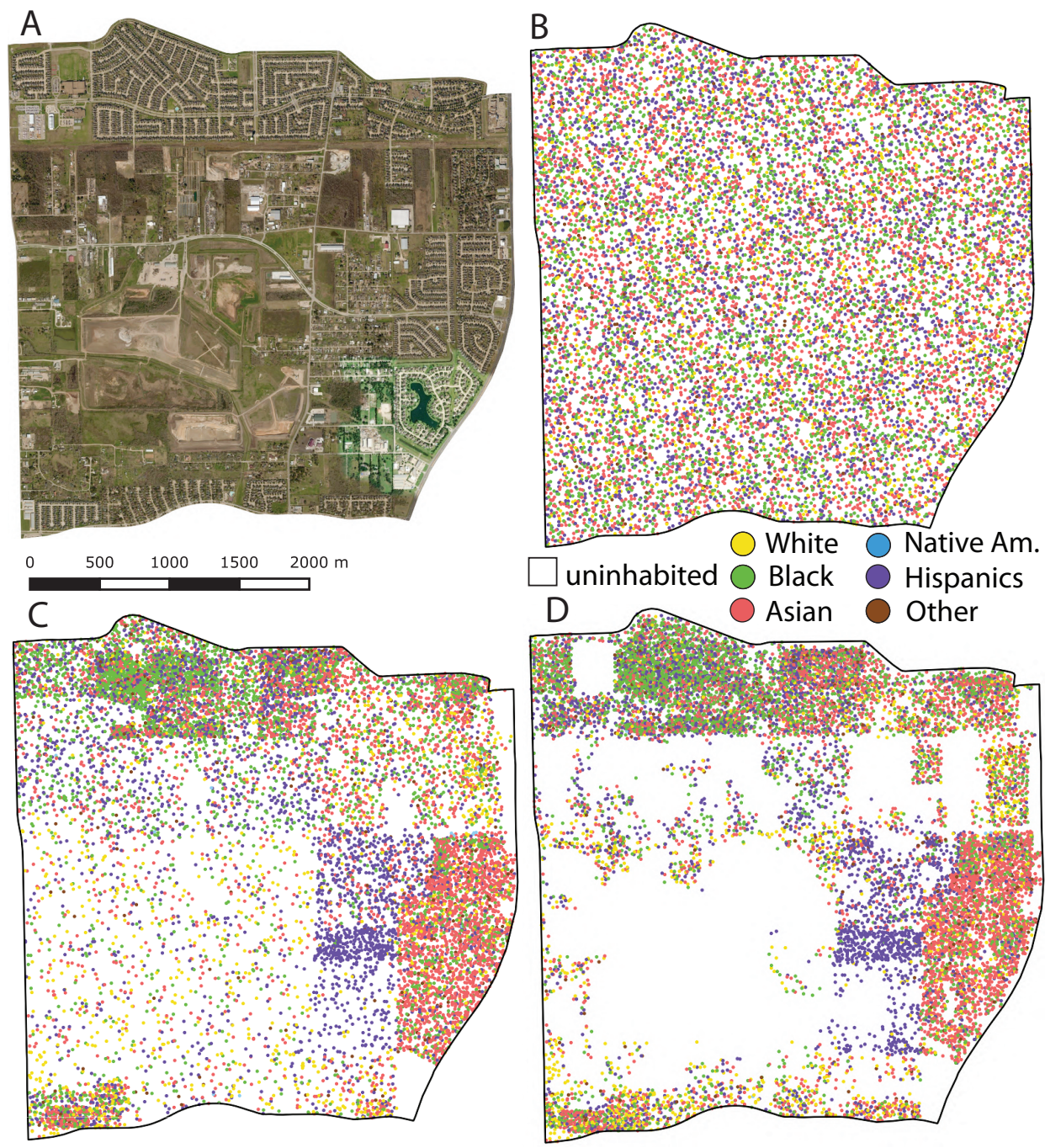

Figure 3. Comparison of racial dot maps of census tract number 6727.01 in Sugar Land, Fort Bend County, TX using different areal units. (A) An aerial image of the tract shows the distribution of inhabited and uninhabited areas. (B) Dot map based on the census tract. (C) Dot map based on census blocks. (D) Dot maps based on grid cells.

prehensive computational environment that includes libraries to work with different types of spatial data. It allows to build efficient, flexible and fully automated computational environment to work with a large dataset. $\mathrm{R}$ script for generating racial dot map from gridded population data is available for download from http://sil.uc.edu. In addition, we have also generated a grid-based racial dot map for each county in the conterminous United States using 2010 census block data and 2011 edition of NLCD. These maps are also available for download from http://sil.uc.edu.

\section{Examples}

Just as using racial dot maps based on census blocks instead of tracts revels subtract structure of segregation (Fowler, 2016), using racial dot maps based on grid cells reveals sub-block structure. These structures are revealed because of the ability of the high-resolution LCLU/NLUD data to distinguish between inhabited and uninhabited areas as well as between areas of different population density. Thus, 

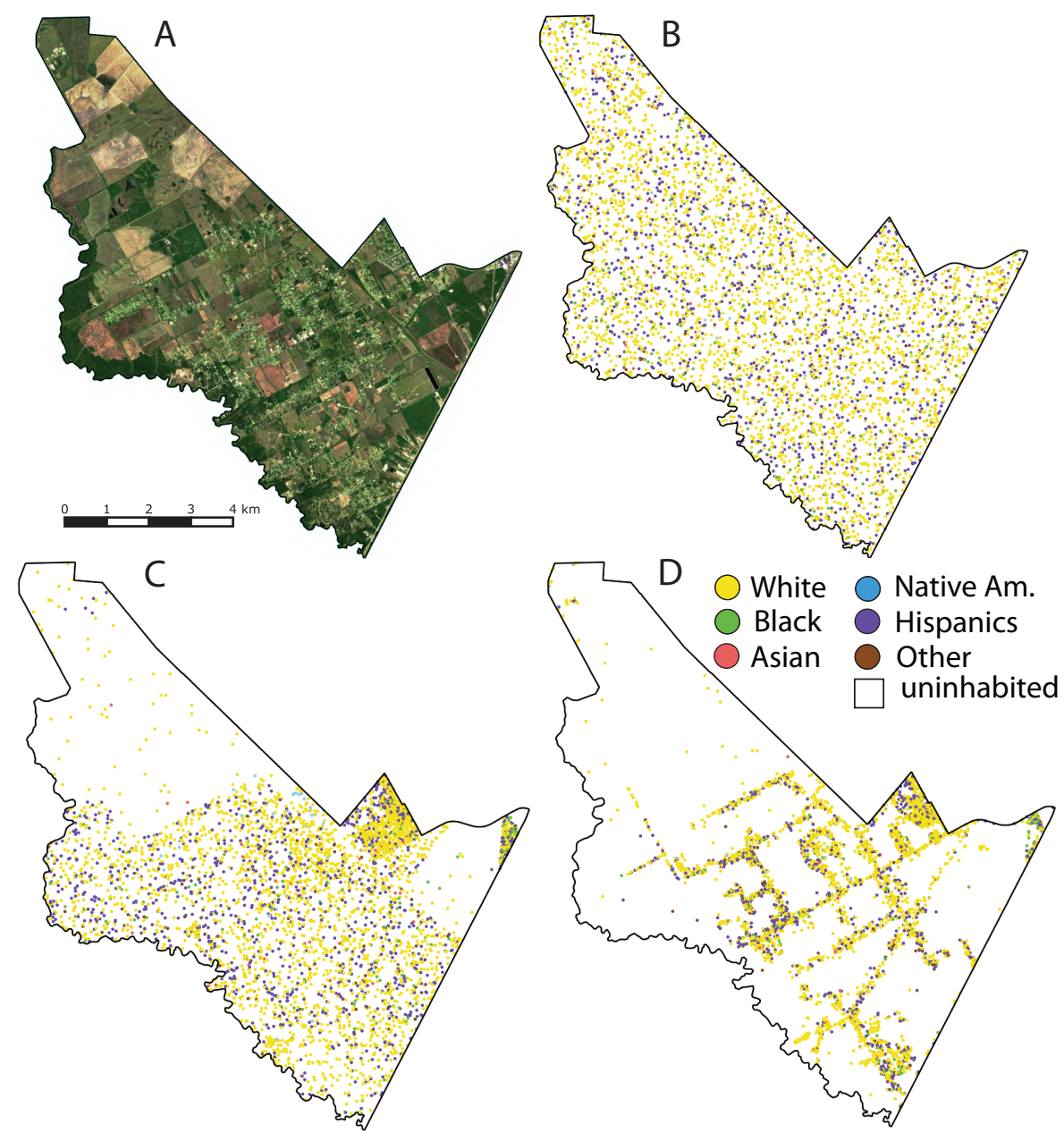

Figure 4. Comparison of racial dot maps of census tract number 6615.02 in Brazoria County, TX using different areal units. (A) An aerial image of the tract shows the distribution of inhabited and uninhabited areas. (B) Dot map based on the census tract. (C) Dot map based on census blocks. (D) Dot maps based on grid cells.

the advantage of grid-based racial dot map manifests itself mostly at small spatial scale. Here we present three examples of how the grid-based dot map provides more information than a block-based dot map. Each example consists of a single census tract. For each tract we show an aerial image for visual reference, a tract-based dot map, a block-based dot map, and a grid-based dot map.

The first example is the tract 6727.01 located in Sugar Land in Fort Bend County, TX. This tract covers an urban environment. Nevertheless, an aerial image of this tract (Figure 3A) indicates the presence of significant sub-block uninhabited areas. A dot map constructed on the basis of the entire tract data (Figure 3B) indicates the presence of a highly diverse population. However, a dot map constructed on the basis of individual blocks data (Figure 3C) shows a high degree of racial segregation. The diversity at the tract level is the result of an arithmetic average of several racially uniform blocks, in reality, people live in communities of low racial diversity. The grid-based map (Figure 3D) does not change the conclusions drawn from the block-based map but provides additional information about the spatial distribution of population by relocating dots from uninhabited areas (compare Figure 3D with Figure 3A) to inhabited areas of blocks. In particular, whereas Figure 3C suggest a presence of large, low density, high diversity region occupying a large portion of the tract, Figure 3D correctly shows that this region is uninhabited.

The second example is the tract 6615.02 in Brazoria County, TX. This tract covers 


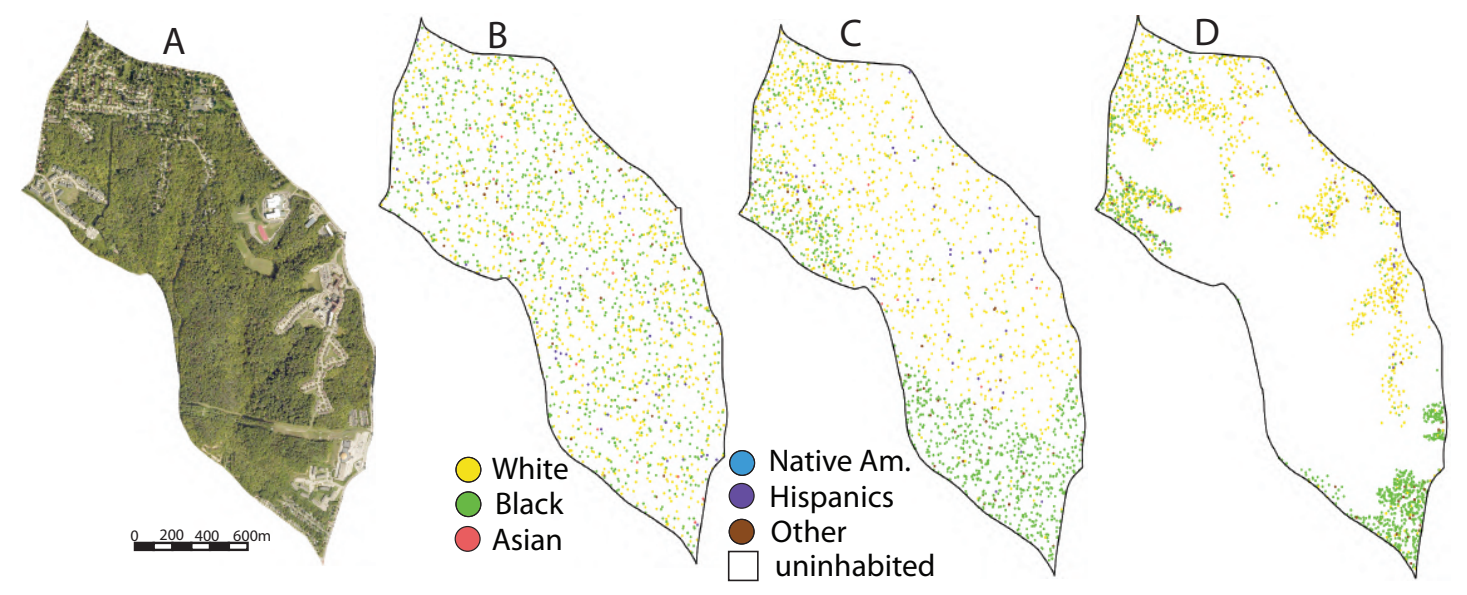

Figure 5. Comparison of racial dot maps of census tract number 008400 in Cincinnati, Hamilton County, OH using different areal units. (A) An aerial image of the tract shows distribution of inhabited and uninhabited areas. (B) Dot map based on the census tract. (C) Dot map based on census blocks. (D) Dot maps based on grid cells.

a rural environment. Its aerial image (Figure 4A) reveals the presence of croplands, with urban structures located only along few roads. As none of that details are available in the tract-level census data, the tract-based racial dot map of this tract (Figure 4B) shows evenly distributed population dominated by whites but with a noticeable presence of Hispanics. Dividing this tract into constituent blocks isolates some of the agricultural and uninhabited areas but not all (Figure 4C). Block-based dot map still incorrectly shows people to be uniformly distributed over blocks having nonzero populations. Grid-based dot map (Figure 4D) shows the correct distribution of population along several roads present in this tract. The between roads areas in blocks with nonzero population are uninhabited agricultural lands.

The third example is the tract 008400 in Cincinnati, Hamilton County, OH. This tract is located in the urban environment but is adjacent to a park as is revealed by its aerial image (Figure 5A), so only portions of the tract are inhabited. Tractbased dot map (Figure 5B) indicates a uniform distribution of population dominated by black residents with some addition of white residents. The block-based map shows a more realistic distribution of population and spatial separation of black and white residents. However, because the block boundaries do not completely separate uninhabited park from inhabited areas, people are still shown to live in what is in reality, a park. The grid-based map (Figure 5D) separates uninhabited and inhabited areas in agreement with the aerial map (Figure 5A). It shows that the majority of the black population lives in the southern part of the tract. Northern parts of this tract are inhabited by a mixture of blacks and whites, whereas eastern parts of the tract are inhabited mostly by whites.

\section{Discussion and Conclusions}

In this paper, we described the construction a grid-based racial dot map and demonstrated that such maps are more spatially accurate than racial dot maps currently in use. We also make available grid-based dot maps for all counties in the conterminous United States and released an R software for calculating dot maps from a grid. This adds to a set of tools that social scientists and others can use to visualize geography of racial segregation and/or diversity. A racial dot map is a visualization tool that supplements analytical approaches to studying racial segregation and diversity of the population. Grid-based racial dot maps are especially useful for visualizing small areas, like, for example, individual tracts.

As we pointed out in the introduction, the number of methods to visualize racial geography is already in use. They can be divided according to two characteristics, a form of the data used and the variable mapped. First, visualizations can be divided 


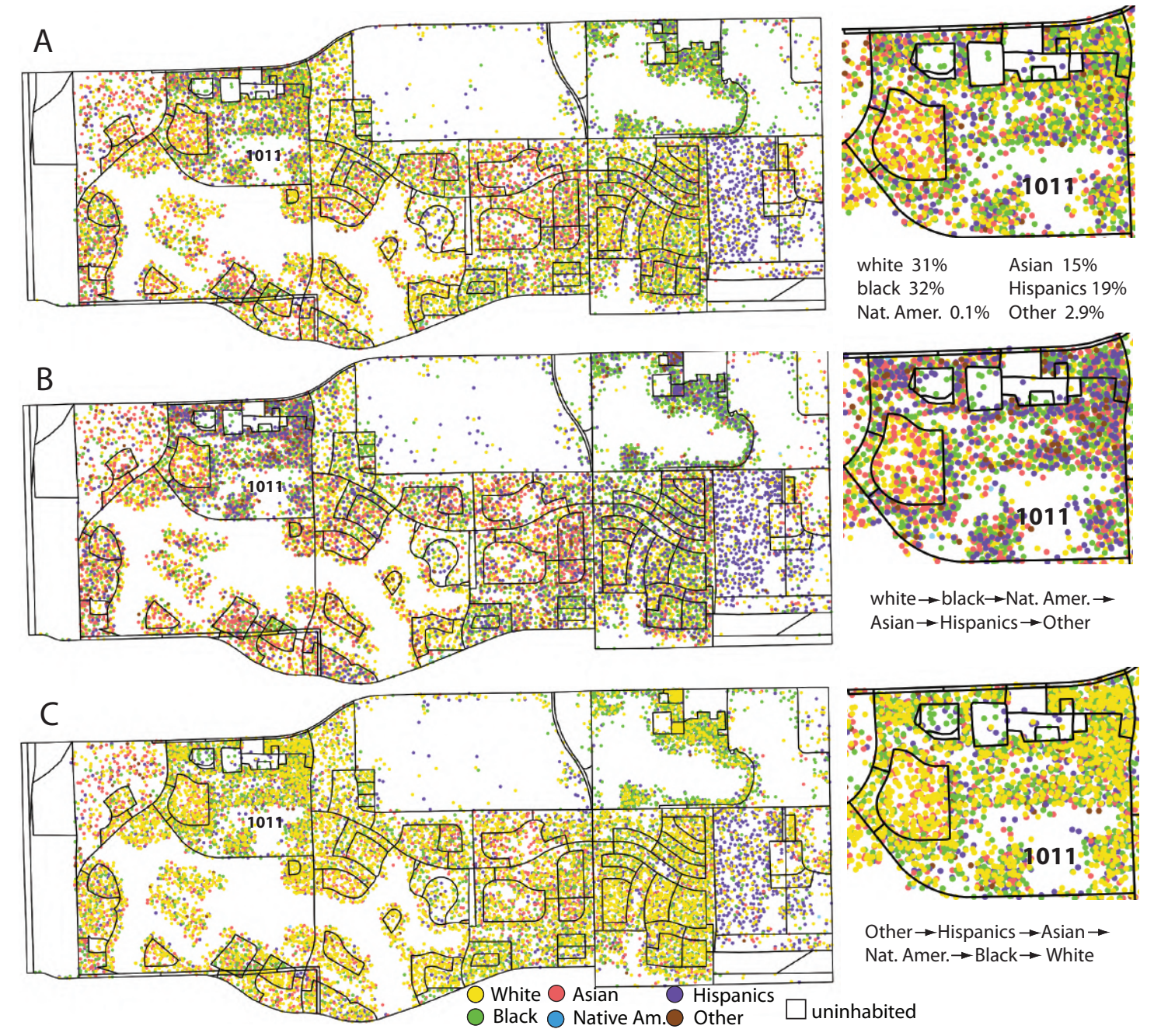

Figure 6. Racial dot maps of the tract 6607.01, Brazoria County, TX constructed using different protocols of placing dots on the map. (A) Dots are placed in random order regardless of the race they represent. (B) Dots are placed sequentially by the race they represent in an indicated order. (C) Dots are placed sequentially by the race they represent in a reversed order. Boundaries of census blocks are indicated by black lines. The left column shows a single block (1011) zoomed in for greater detail.

according to the form of data used - either the original census data or gridded data. Using original census data yields a choropleth map with a resolution corresponding to the size of selected census units. In the United States, tracts are the most often used areal units and blocks offer the highest spatial resolution. An average census tract has a population size of 4,000 people, however, the spatial size of census tracts varies widely depending on population density. A census block is the smallest geographic unit used by the U.S. Census Bureau. The population of census blocks varies from 0 to several hundred. On the other hand, the SocScape gridded data is raster of individual cells, having the size $30 \mathrm{~m} \times 30 \mathrm{~m}$, storing information about the local population density. Because in social science the census data is a recognized standard, the majority of visualization of racial geography is based on tracts and some are based on blocks. However, grids are freely available (at least for the United States) so there is no barrier for their use in visualizing racial geography where high spatial resolution is needed.

Second, visualizations can be divided according to a variable mapped. Percentage of people of a given race is a variable traditionally used to study racial geography. Web apps such as Census Data Mapper (https://www.census.gov/geo/maps-data/maps/datamapper.html) or Social 
Explorer (https://www.socialexplorer.com/) can be used to visualize a spatial distribution of a percentage of a given race at the resolution of census units. The grid-based equivalent of such maps can be constructed using data available at http://sil.uc.edu. Better visualization of racial geography is provided by maps which use diversity/dominant race categories. Samples of such maps are shown in Figure $1 \mathrm{~B}$ and E. Census tract-based racial classification maps can be explored on the Mixed Metro website (http://mixedmetro.com/), and grid-based racial classification maps can be explored on the SocScape website (http://sil.uc.edu/webapps/socscape_usa/).

Even better visualization of racial geography is provided by racial dot maps. The advantage of a dot map over the racial classification map is that it explicitly shows the racial mix, whereas presently used categories concentrate on a level of diversity and indicate only a dominant race. Of course, adding more categories to a racial classification map could address this problem but the map would become difficult to read. The disadvantage of a dot map is that it can only be used for visualization whereas a classification map is a visualization and a dataset which can be used for quantitative analysis.

The significance of this work is that it presents a method of constructing racial dot maps from dasymmetrically disaggregated data instead of aggregated data. This distinguishes the present method from all previous works and allows it to visualize more accurately racial distribution within small areas, such as, for example, census tracts. The advantage comes from using a dasymetric population data model which utilizes additional data (land cover) to prevent placing people within uninhabited regions. An additional contribution is the use of random order of placing dots regardless of their labels. This technique results in a more correct visualization of a racial mix in densely populated areas.

To illustrate the importance of random order placing of dots Figure 6 shows a racial dot map of tract number 6607.01 (located in Brazoria County, TX) constructed three times. The first time a map is constructed with random order placement of dots (Figure 6A), The second time a map is constructed by placing dots for different races in order as indicated in Figure 6B. The third time a map is constructed by placing dots in reverse order (Figure 6C). In the densely populated areas of the tract a non-random placement orders (panels B and $\mathrm{C}$ ) result in erroneous (and different from each other) interpretation of a racial mix. Zooming into a single block (right column in Figure 6) reveals that ordered placement leading to the map in Figure 6B indicates the dominance of Hispanics, whereas the reverse placement, leading to a map shown in Figure 6C, indicates domination of whites. In reality, the population in this block is very diverse with no dominant race (see percentages of each race listed in Figure 6). This actual mixture is well reflected by placing dots in random order regardless of the race they represent. This may seem like an obvious design element of any racial dot map, but many published dot maps (for example the University of Virginia map) employ sequential placing and thus may not correctly reflect a true racial mix in densely populated areas.

Visual analysis of dot maps vis-a-vis an aerial image in examples given in section 3 clearly shows that in comparison to a block-based dot map the grid-based map provides a more realistic depiction of the spatial distribution of population while preserving information about racial diversity. Dot maps based on tracts should be avoided because they may lead to false conclusions about levels of racial segregation and/or diversity.

\section{References}

Bader, Michael D.M and Siri Warkentien. 2016. The fragmented evolution of racial integration since the Civil Rights Movements. Sociological Science 3, 133-166.

Clark, W. A., E. Anderson, J. Östh, and B. Malmberg. 2015. A multiscalar analysis of neighborhood composition in Los Angeles, 2000-2010: A Location-Based Approach to segregation and diversity. Annals of the Association of American Geographers 105(6), 1260-1284.

Dmowska, Anna and Tomasz F Stepinski. 2014. High resolution dasymetric model of U.S. demographics with application to spatial distribution of racial diversity. Applied Geography 53, 417-426.

Dmowska, Anna and Tomasz F Stepinski. 2016. Mapping changes in spatial patterns of racial 
diversity across the entire United States with application to a 1990-2000 period. Applied Geography 68, 1-8.

Dmowska, Anna and Tomasz F Stepinski. 2017a. A high resolution population grid for the conterminous United States: The 2010 edition. Computers,Environment and Urban Systems 61, $13-23$.

Dmowska, Anna and Tomasz F Stepinski. 2017b. Mapping changes of racial composition in the United States: 1990-2010. In Population Association of America 2017, Chicago, April 26-29 2017. https://paa.confex.com/paa/2017/meetingapp.cgi/Paper/10564.

Dmowska, Anna, Tomasz F Stepinski, and Pawel Netzel. 2017. Comprehensive framework for visualizing and analyzing spatio-temporal dynamics of racial diversity in the entire United States. PLoS ONE 12(3), e0174993.

Farrell, Chad R. 2008. Bifurcation, fragmentation or integration? The racial and geographical structure of US Metropolitan segregation, 1990-2000. Urban Studies 45(3), 467-499.

Farrell, Chad R and Barrett A Lee. 2011. Racial diversity and change in metropolitan neighborhoods. Social Science Research 40(4), 1108-1123.

Fasenfest, David, Jason Booza, and Kurt Metzger. 2004. Living together: A new look at racial and ethnic integration in metropolitan neighborhoods, 1990-2000. Technical report.

Fowler, Christopher S. 2016. Segregation as a multiscalar phenomenon and its implications for neighborhood-scale research: the case of South Seattle 1990-2010. Urban Geography 37(1), $1-25$.

Frey, William H and Reynolds Farley. 1996. Latino, Asian, and black segregation in U.S. metropolitan areas: Are multiethnic metros different? Demography 33(1), 35-50.

Frey, William H and Dowell Myers. 2005. Racial segregation in US metropolitan areas and cities, 1990-2000: Patterns, trends, and explanations. Technical report.

Holloway, Steven R, Richard Wright, and Mark Ellis. 2012. The racially fragmented City? Neighborhood racial segregation and diversity jointly considered. The Professional Geographer $64,63-82$.

Iceland, John. 2004. Beyond Black and White metropolitan residential segregation in multiethnic America. Social Science Research 33, 248-271.

Iceland, J, DH Weinberg, and E Steinmetz. 2002. Racial and ethnic segregation in the United States: 1980-2000 (Census 2000 Special Report Series CENSR-3). Washington, DC: US Census Bureau.

Johnston, R., M. Poulsen, and J. Forrest. 2007. Ethnic and racial segregation in U.S. metropolitan areas, 1980-2000: The dimensions of segregation revisited. Urban Affairs Review 42(4), 479-504.

Kimerling, A. Jon. 2009. Dotting the dot map, revisited. Cartography and Geographic Information Science 36(2), 165-182.

Lichter, Daniel T, Domenico Parisi, Steven Michael Grice, and C Taquino. 2007. National estimates of racial segregation in rural small-town America. Demography 44(3), 563-581.

Logan, John R. 2015. Chalanges of spatial thinking. In F. M. Howell, J. R. Porter, and S. A. Matthews (Eds.), Recapturing Space: New Middle-Range Theory in Spatial Demography, Chapter 2, pp. 11-36. Springer.

Logan, John R, Brian J Stults, Reynolds Farley, and J Stults. 2004. Segregation of minorities in the metropolis: Two decades of change. Demography 41(1), 1-22.

Massey, Douglas S and Nancy A Denton. 1987. Trends in the residential segregation of Blacks, Hispanics, and Asians: 1970-1980. American Sociological Review 52(6), 802-825.

Petrov, Andrey. 2012. One hundred years of dasymetric mapping: Back to the origin. Cartographic Journal 49(3), 256-264.

R Core Team. 2014. R: A Language and Environment for Statistical Computing. Vienna, Austria: R Foundation for Statistical Computing.

Seguin, Ch, A Nierobisz, and K.P Kozlowski. 2017. Seeing race: teaching residential segregation with the racial dot map. Teaching Sociology 45(2), 1142-151.

Sperling, J. 2012. The tyranny of census geography: Small-area data and neighborhood statistics. Cityscape 14(2), 219-223. 
Theobald, David M. 2014. Development and applications of a comprehensive land use classification and map for the US. PloS one 9(4), e94628.

Wong, David. 2015. From Aspatial to Spatial, from global to local and individual: Are we on right track to spatialize segregation measures? In F. M. Howell, J. R. Porter, and S. A. Matthews (Eds.), Recapturing Space: New Middle-Range Theory in Spatial Demography, Chapter 5, pp. 77-98. Springer.

Wright, R, M Ellis, S.R Holloway, and S Wong. 2014. Patterns of racial diversity and segregation in the United States: 1990-2010. The Professional Geographer 66 (2), 173-182. 